\title{
Here comes the neighborhood
}

Laser-guided assembly of protein microscaffolds lets researchers construct multispecies cellular communities.

Bacteria can band together for both good and ill, with different species exchanging chemical signals that modulate each other's growth, metabolism and interactions with the surrounding environment. Conventional culture systems are of limited value in attempting to capture this complexity, but research from chemist Jason Shear and microbiologist Marvin Whiteley of the University of Texas at Austin demonstrates an elegant microfabrication strategy that adds another dimension to bacterial research.

Shear's group uses lasers for the precision crafting of microstructures from cross-linked proteins via a process known as multiphoton lithography (MPL), which makes it possible to observe the behavior of cells within custom-built three-dimensional (3D) environments. "Unlike plastic or glass or silicon, these materials are highly porous and are not going to trap chemicals along with the cells," says Shear. "They're going to allow nutrients to diffuse through, and signals that cells release can diffuse out."

Shear's group has now extended this technique to enable 'laser printing' of multispecies bacterial communities. After diluting microbes in a solution of melted gelatin containing the protein bovine serum albumin (BSA) and a photosensitizing compound, they allow the mixture to cool and solidify. The sample is then subjected to MPL, wherein a laser is focused at precise points within the gelatin matrix, activating the photosensitizing agent and promoting cross-linking of the gelatin and BSA into a rugged but porous protein shell that can be constructed with submicrometer resolution. The gelatin can then be melted and washed away, leaving encapsulated cells behind to procreate within their protein prison. By performing this process in multiple rounds, the researchers can build sophisticated layered architectures that combine multiple cell types in tightly controlled arrangements.

Whiteley's team has long been interested in understanding how interactions among different pathogenic bacteria can affect the severity of infections.. He and Shear initially tested this technique with a combination of Staphylococcus aureus and Pseudomonas aeruginosa. "Pseudomonas is a major cause

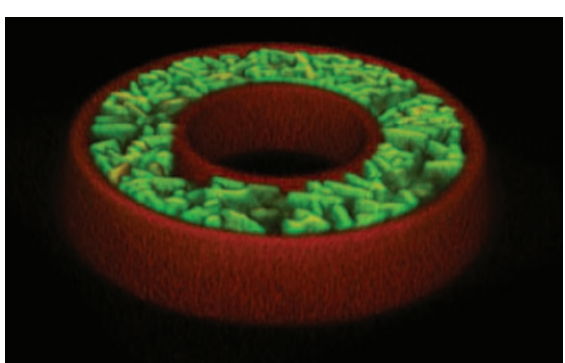

Precisely targeted laser cross-linking generates 3D cellular habitats, as with this toroid full of Pseudomonas bacteria. Image courtesy of J. Shear.

of infections in the lungs of cystic fibrosis patients," says Shear, "but it's not only Pseudomonas-it's a polymicrobial infection where Staphylococcus is often another major player." These two cell types are difficult to cocultivate: Staphylococcus adheres to solid surfaces, whereas Pseudomonas is generally free swimming until it encounters conditions that favor formation of dense biofilms. Using MPL, however, Shear and colleagues could contain Staphylococcus within a 'core' surrounded by Pseudomonas cells encased by an additional 'shell' layer. Both types of bacteria thrived within their respective environments, but this arrangement also conferred an additional benefit on Staphylococcus: a big boost in resistance to the antibiotic ampicillin. This effect was even more pronounced when the outer shell contained ampicillin-resistant Pseudomonas, which increased the survival rate of ampicillin-treated Staphylococcus to $80 \%$. For Staphylococcus cultivated within a core scaffold alone, the survival rate was only $\sim 20 \%$.

The researchers hope to further explore the relationship between these bacteria in other pathogenic settings and recently received a grant from the Army Research Office to study how this interplay might contribute to microbial resistance to treatment in chronic wounds. Shear is also interested in modeling cellular interactions within human tissues, such as how cancer cells interact with surrounding healthy tissues. "We're kind of like kids in a candy store right now," he says, "and I think there are a lot of exciting possibilities for interacting with different collaborators." Michael Eisenstein

\section{RESEARCH PAPERS}

Connell, J.L. et al. 3D printing of microscopic bacterial communities. Proc. Natl. Acad. Sci. USA doi:10.1073/pnas.1309729110 (7 October 2013). 